\title{
Impact of a Central Line Infection Prevention Bundle in Newborn Infants
}

\author{
Rowena McMullan, FRACP; ${ }^{1,2}$ Adrienne Gordon, $\mathrm{PhD}^{1,2}$
}

\begin{abstract}
ов јестіле. To compare central line use and central line-associated bloodstream infection in newborn infants before and after the introduction of a central line infection prevention bundle in order to determine the effectiveness of the bundle and to identify areas for further improvement.
\end{abstract}

DESIGN. Retrospective cohort analysis of prospectively collected data.

Setting. Level 5 neonatal intensive care unit in Sydney, Australia.

patients. Newborn infants admitted to the Royal Prince Alfred Hospital Neonatal Intensive Care Unit who had a central venous catheter (CVC) inserted.

METHODs. Data regarding clinical characteristics, CVC use, and infection were collected before and after the introduction of a bundle of interventions. The bundles encompassed (1) insertion of CVC, (2) maintenance of CVC, (3) an education program, and (4) ongoing surveillance and feedback.

RESULTS. Baseline and intervention groups were comparable in clinical characteristics. The number of CVCs inserted was reduced in the intervention group (central line utilization rate, 0.16 vs $0.2, P<.0001$ ). Overall CVC dwell time was reduced, resulting from significant reduction in peripherally inserted CVC dwell time (6 days [95\% CI, 5.0-11.8 days] vs 7.3 days [4.0-10.4 days], $P=.0004$ ). Central lineassociated bloodstream infections were significantly reduced, predominantly secondary to decreased peripherally inserted CVC-related bloodstream infections $(1.2 / 1,000$ central line-days vs $11.5 / 1,000$ central line-days, $P<.0001)$.

CONCLUsion. This central line infection bundle was effective in reducing CVC use, dwell time, and central line-associated bloodstream infections.

Infect Control Hosp Epidemiol 2016;3 7:1029-1036

Safe and reliable vascular access is a necessary part of care for sick infants in the neonatal intensive care unit (NICU). Central venous catheters (CVCs) are commonly used to provide longterm venous access. They provide critical nutrition for growth as well as a portal for other vital intravenous fluids and medications. They provide improved nutrition and avoid repeated painful procedures associated with the use of peripheral venous cannulas. ${ }^{1,2}$ CVCs used in the NICU include umbilical venous catheters (UVCs) and peripherally inserted CVCs (PCVCs). PCVCs are inserted via a peripheral vein and threaded so that the tip of the catheter lies in a large central vein. UVCs also have the tip sited in a large central vein but are inserted via the umbilical vein.

There are a number of complications associated with CVC use, varying from minor and easily treatable to life-threatening. The most frequent complication is central line-associated bloodstream infection (CLABSI). The Centers for Disease Control and Prevention defines a CLABSI as "a primary blood stream infection in a patient that had a central line within the 48-hour period before the development of the blood stream infection, and is not related to an infection at another site." 3 The incidence of CLABSI varies widely within the population studied and definition used, but is $1.6-15$ per 1,000 central line-days in NICUs in high income countries. ${ }^{4-6}$ Incidence increases with decreasing birthweight and gestational age. ${ }^{7}$ Infection usually occurs via skin commensals that migrate via the catheter entry site or cannula hub, the predominant causative organism being coagulase-negative Staphylococcus. ${ }^{8,9}$ The umbilical stump is particularly heavily colonized, but a recent retrospective cohort analysis demonstrated that UVC CLABSI rate is similar to that in PCVC. ${ }^{10}$ CLABSI is responsible for $69 \%$ of all late-onset infections in preterm babies. ${ }^{11}$ Late-onset neonatal sepsis is a significant risk factor for increased mortality and prolonged hospital stays, ${ }^{12}$ although mortality is variable and related to the implicated pathogen. ${ }^{13}$ In those who survive, there is poorer long-term

Affiliations: 1. Department of Newborn Care, Royal Prince Alfred Hospital Women and Babies, Sydney, Australia; 2. Charles Perkins Centre, University of Sydney, Sydney, Australia.

Received February 23, 2016; accepted May 8, 2016; electronically published June 13, 2016

(c) 2016 by The Society for Healthcare Epidemiology of America. All rights reserved. 0899-823X/2016/3709-0004. DOI: 10.1017/ice.2016.127 
growth and developmental outcomes, ${ }^{14,15}$ with associated increased morbidity and increasing healthcare costs. ${ }^{16}$

Prevention of CLABSI is a key objective for improvement of patient safety and reduction of mortality, hospital stay, and costs. Preventing and controlling healthcare-associated infection is one of the 10 Australian National Safety and Quality Health Service standards, highlighting the national commitment to these preventable infections. ${ }^{17}$ Fortunately, CLABSI has been shown to be highly modifiable with "bundles" of health care interventions. ${ }^{18,19}$ A bundle is defined as "a limited number of specific practices, each essential for effective and safe patient care and that, when implemented together, result in additional improvements in patient outcomes." ${ }^{20}$ These multidisciplinary, evidence-based best practice recommendations are effective in reducing CLABSI in the NICU. ${ }^{20,21}$

An audit of CLABSI rates in 2012 at our institution ${ }^{22}$ showed 8.5 CLABSI/1,000 central line-days, and 13.4/1,000 central linedays for infants less than 29 weeks' gestation. Comparative data combining rates from all the other level 5 NICUs in New South Wales over the same period using the same definition of CLABSI showed a CLABSI rate of 8.3/1,000 central line-days at less than 29 weeks' gestation. ${ }^{23}$ The audit identified a number of areas for improvement: the implementation of standardized practices, central line policy revision, and a structured education program. Following this, a bundle of CLABSI prevention interventions was introduced. The aim of this study was to compare CLABSI rates before and after the introduction of the CLABSI prevention bundle to determine its effectiveness and to identify areas for further improvement.

\section{METHODS}

\section{Study Setting and Design}

This was a retrospective cohort analysis of prospectively collected data. Eligible infants were admitted to the Royal Prince Alfred
Hospital NICU and had a CVC inserted. The study periods were January 1, 2012-December 31, 2012 (baseline) and August 1, 2013-July 31, 2014 (intervention). The bundle of interventions commenced during March-August 2013.The intervention period was chosen as the most recent fully audited data available. This hospital is a major obstetric tertiary referral center. The hospital currently averages approximately 5,500 deliveries per year and covers an inner-city, multicultural population. It provides a level $5 \mathrm{NICU}$ service $^{24}$ with an average of approximately 900 admissions to the nursery each year. This study was approved prospectively by the Sydney Local Health District ethics committee.

\section{Process and Interventions}

Following the audit, a multidisciplinary team of staff with an interest in quality improvement and infection control was formed. Areas where CLABSI prevention had failed were reviewed, and solutions were proposed. These were based upon current best evidence, and largely upon the recommendations from the Provincial Infectious Diseases Advisory Committee, Ontario. ${ }^{25}$ A fishbone diagram was used to highlight the issues (Figure 1).

The baseline and intervention central line practices are documented in Table 1. It must also be noted that probiotics were introduced into routine newborn care from October 2012 for all infants less than 30 weeks' gestation, or greater than 29 weeks' gestation with additional risk factors placing them at higher risk for necrotizing enterocolitis. There were no other relevant changes in policy or definitions during the study periods.

\section{Implementation Strategy}

The bundle of interventions was implemented via a structured education program. There is a strong background within this

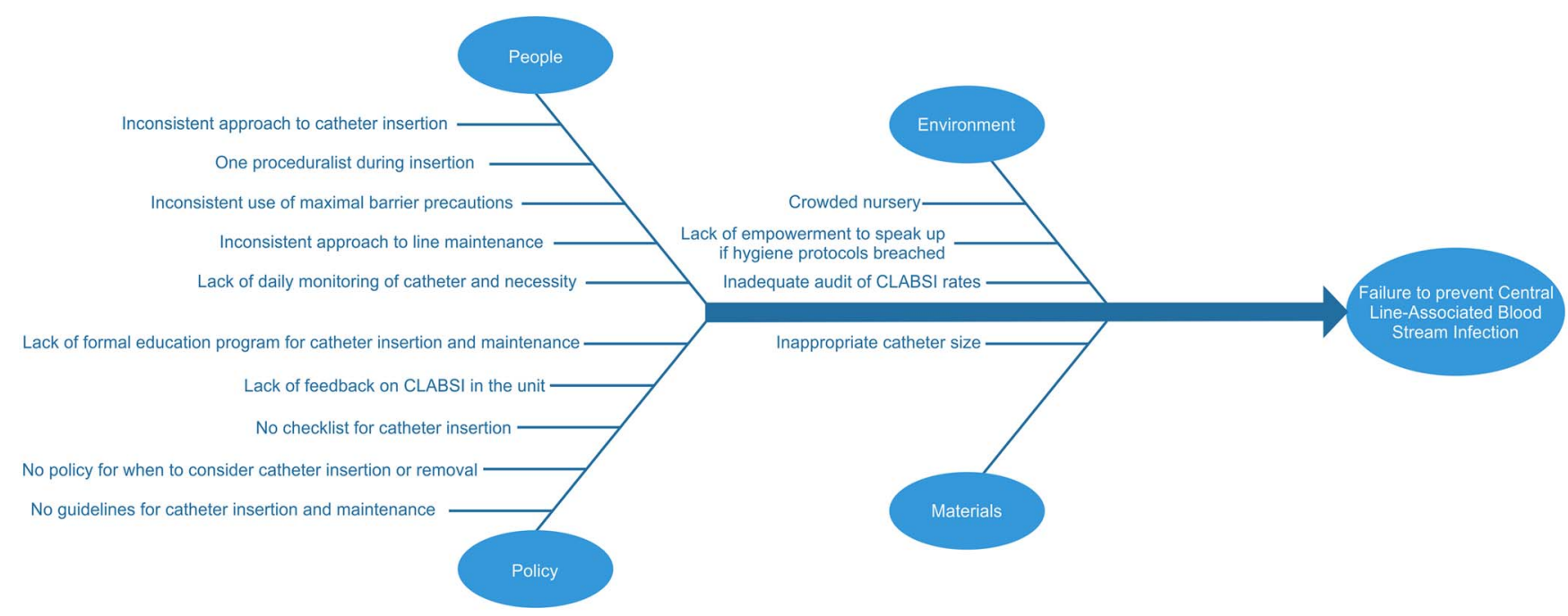

FIGURE 1. Fishbone diagram highlighting process failures in prevention of central line-associated bloodstream infection (CLABSI). 


\section{Insertion bundle}

No formalized CVC discussions.

CVC left in situ for 24 hours after full feeds reached at $150 \mathrm{~mL} / \mathrm{kg} / \mathrm{day}$.

If TPN indicated, CVC placed if likely to require TPN for more than 5 days.

Inconsistent approach to sterile precautions.

Only 1 person scrubbed.

No hat or mask worn.

Procedure performed through doors of the incubator.

No isolation of bed space.

Inconsistent choice of CVC or site of insertion.

Supervision recommended but not mandated in policy.

Clean twice with aqueous $0.015 \%$ chlorhexidine and allow to air dry for 3 minutes.

Practitioner makes entry into medical records after the procedure. Inconsistent details recorded.

Maintenance bundle

Central line stop cocks/ hubs (eg, medication). Sealed nutrition and bags and use of 3-way taps. 48-hour TPN. Minimize line breaks. Maximum sterile precautions for line breaks.

Scrub the hub for $15-30$ seconds with chlorhexidine $0.5 \%$ solution and allow to dry.

Dressing replaced when soiled or integrity compromised.

Surveillance bundle

Inconsistent hand hygiene monitoring with minimal feedback.

Infections coded by staff specialists ad hoc.

Attendance at some ward rounds of external infectious diseases specialist.

Annual feedback from statewide data, provided $>1$ year after data collected.

Irregular audit.

Education bundle

Ad hoc teaching provided 3-6 times/ month. Different educators would utilize different teaching styles and materials and focus on different topics within CVC use.
Daily discussions as part of clinical handover regarding CVC necessity. ${ }^{a}$

Removal of CVC at earliest opportunity.

Use of maximal sterile precautions including use of hat, mask, double glove, and gown for anyone within $1 \mathrm{~m}$ of sterile field.

Isolating bed space with a screen.

3-minute WHO surgical hand scrub before gowning.

2 people scrubbed per line insertion.

Draping of entire patient, using open care or incubator with side down.

2-Fr CVC as preferred access. 1-Fr only for $<1 \mathrm{~kg}$, or if unable to insert a larger CVC.

Sites of preference: lower limb $>$ upper limb > scalp.

Inexperienced staff to be directly supervised at all times. Only senior staff to insert CVC in infants $<750 \mathrm{~g}$.

Unchanged.

Use of a checklist and sticker to add to medical records.

Unchanged.

If dressing compromised then replaced if appropriate under full sterile precautions. Otherwise CVC removed.

Observed moments of hand hygiene with quarterly feedback to all staff.

Monthly infection meeting of all senior staff with hospital microbiologist to discuss and code infection data.

Weekly attendance on ward rounds of local infectious diseases specialist.

Real-time monthly feedback of CLABSI rates to senior staff. Quarterly feedback provided to all staff via education program.

Second yearly audit and formal report detailing CVC use and CLABSI rates.

Structured and formalized education program, based on the SCORPIO method of teaching ${ }^{26}$ and covering CVC use, insertion, maintenance, and prevention of CLABSI.

Includes formative assessment and evaluation. Consistent education materials prepared in advance.

NOTE. CLABSI, central line-associated bloodstream infection; CVC, central venous catheter; Fr, French; SCORPIO, Structured, Clinical, Objective Referenced, Problem-based,

Integrated, and Organized; TPN, total parenteral nutrition; WHO, World Health Organization.

${ }^{a}$ Daily discussion included both initial decision to insert a CVC as well as ongoing requirement for it to remain in situ. There was a concerted effort to avoid CVC in larger and mature infants likely to tolerate enteral nutrition well. 
unit with the SCORPIO ${ }^{26}$ (Structured, Clinical, Objective Referenced, Problem-based, Integrated, and Organized) method of teaching. This approach uses interactive problemfocused learning to teach specific skills and has been adapted for adult postgraduate learning. ${ }^{27}$ It was the successful foundation of the SEA-URCHIN Project ${ }^{28}$ (South East Asia-Using Research for Change in Healthcare-Associated Infections) that was also initiated by this unit, with which our bundle had similar objectives and some shared materials. Education sessions were conducted every 3 months, covering all new staff and providing updates to all staff at least yearly. There were 3 workshops, each lasting 1.5-2.5 hours and containing an introduction, a number of skills-focused stations, an objective structured clinical examination, and formative assessment. Each workshop had a coordinator and a number of facilitators, who were medical staff and nurse educators with an interest in infection control. The central line workshop contained an introduction with an overview of CLABSI and 4 stations: (1) preparation to handle or insert lines, (2) CVC insertion, (3) CVC maintenance, and (4) local and state audit feedback of CLABSI. The infection control workshop consisted of 4 stations: (1) hand hygiene, (2) infection control at birth, (3) nursery admission, and (4) nursery environment. A hand hygiene workshop consisted of an introduction with local audit feedback and 3 stations: (1) the World Health Organization's 5 Moments for Hand Hygiene, ${ }^{29}$ (2) effective hand hygiene, and (3) assertiveness training.

\section{Data Collection}

Information was identified and obtained from the neonatal clinical database. Data were extracted for every infant who had a CVC placed during his or her NICU admission. If further clarification was required, there was validation of data with the full medical record. Data regarding clinical characteristics, CVC use, and details of any bloodstream infection were collected. Infections are coded monthly by a committee of attending neonatologists and the hospital microbiologist using the following definitions.

\section{Definitions}

Central line was defined as a venous line inserted via either the umbilical or a peripheral vein, such that the line tip is placed into a large central venous vessel. ${ }^{30}$ Central lines included UVC and PCVC.

Proven bloodstream infection was defined as growth of a certain pathogen in blood and treated by the clinicians as infection. If the pathogen could be a potential contaminant, there must be a pure growth of that organism, with either confirmatory laboratory evidence or growth of the same organism on repeat culture.

CLABSI was defined as a proven bloodstream infection associated with a central venous line when a central line has been in use 48 hours before signs and symptoms of an infection with no apparent source other than the central line. A culture of the same organism in any sample within 13 days is counted as a single infection. This definition was unchanged throughout the studied periods.

Early-onset infection was defined as a proven bloodstream infection with initial symptoms occurring sooner than 48 hours after birth.

Late-onset infection was defined as a proven bloodstream infection with initial symptoms occurring at least 48 hours after birth.

Babies were analyzed as part of gestational cohort groups. These groups included less than 29, 29-31, 32-36, and at least 37 weeks' gestation.

\section{Statistical Analysis}

Data were managed with an electronic spreadsheet (Excel 2013; Microsoft) and analyzed using Prism, version 5.0 for Windows (GraphPad). The central line utilization ratio was calculated as the number of central line-days/number of patient-days. CLABSI rates were calculated as number of CLABSI/central line-days $\times 1,000 .^{7}$ Continuous data were expressed as mean (SD) or median (interquartile range) according to the distribution. Data were compared with the Mann-Whitney test or the unpaired $t$ test as appropriate. Categorical data were expressed as count and proportion and compared with the $\chi^{2}$ or Fisher exact test. One-way analysis of variance (Kruskal-Wallis test) was used to compare more than 2 groups. All reported $P$ values are 2 sided, and significance was assumed at $P<.05$.

\section{RES U L T S}

\section{Clinical Characteristics}

Patient demographic characteristics are shown in Table 2. There were no significant differences between the groups in any of the parameters recorded.

\section{Central Line Use}

With regard to central line insertion, 353 CVCs (177 UVCs and 176 PCVCs) were inserted in 214 newborns during the baseline period, compared with 260 CVCs (142 UVCs and 118 PCVCs) inserted in 162 newborns during the intervention period. There were significantly fewer CVC inserted in the intervention period; the central line utilization rate was 0.11 in the intervention period vs 0.2 at baseline (Fisher exact test, $P=.0001$ ).

Overall, median dwell time of CVCs was significantly shorter in the intervention period compared with the baseline period; $4.4(95 \% \mathrm{CI}, 2.2-6.7)$ vs $5.0(2.9-8.2)$ days (MannWhitney test, $P=.01)$. A reduction in PCVC dwell time underlies this difference: $6(5.0-11.8)$ vs $7.3(4.0-10.4)$ days (Mann-Whitney test, $P=.0004$ ) (Figure 2). UVC dwell times were not significantly different in intervention and baseline 
Tав ве 2. Characteristics of Infants With Central Lines Inserted

\begin{tabular}{lccr}
\hline Characteristic & Baseline $(n=214)$ & Intervention $(n=162)$ & $P$ \\
\hline Male sex & $134(63)$ & $95(59)$ & .46 \\
Gestation, median (IQR), weeks & $32(23-41)$ & $31(24-41)$ & .52 \\
Birthweight, median (IQR), g & $1,660(1,155-2,781)$ & $1,644(1,176-2,530)$ & .85 \\
Singleton & $176(82)$ & $126(78)$ & .30 \\
Antenatal steroids $<37$ weeks & $143 / 165(87)$ & $117 / 126(93)$ & .12 \\
Delivery by Caesarean & $130(61)$ & $110(68)$ & .16 \\
Chorioamnionitis & $65(30)$ & $62(38)$ & .12 \\
Ex-utero transfer & $34(16)$ & $29(18)$ & .68 \\
\hline
\end{tabular}

NOTE. Data are no. (\%) of infants unless otherwise indicated. IQR, interquartile range.

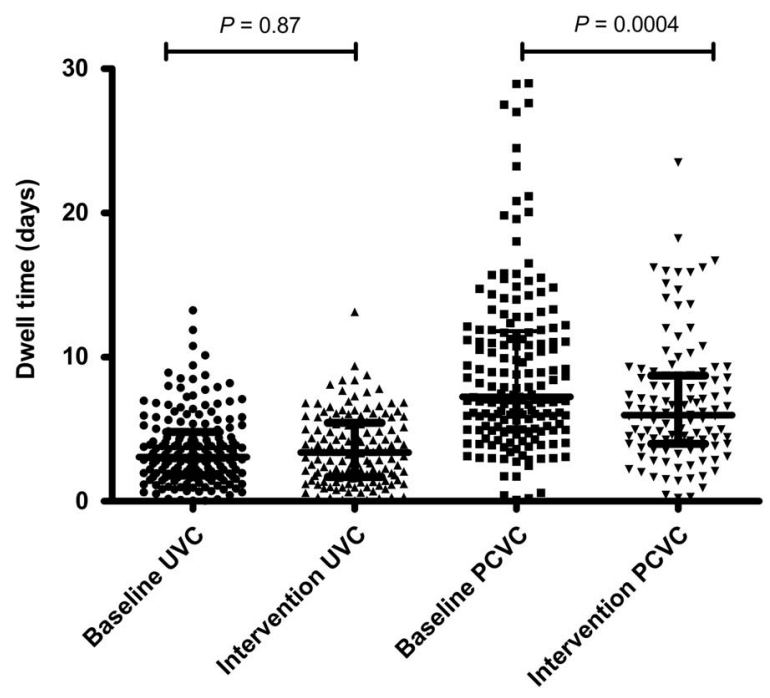

FIGURE 2. Dwell time of central venous catheters in the baseline and intervention groups. PCVC, peripherally inserted central venous catheter; UVC, umbilical venous catheter.

groups (3.1 [1.8-4.8] vs 3.4 [1.7-5.4], $P=.87)$. Dwell time was not different in babies less than 31 weeks' gestation (5.9 [2.910.4] vs 5.5 [2.9-7.8] days, $P=.19$ ) but was significantly shorter for those babies at least 31 weeks' gestation in the intervention period (3.7 [1.9-5.5] vs 4.2 [2.8-6.8] days, $P=.05)$. Dwell time was longer in preterm infants in comparison with term and was inversely proportional to gestational age ( 1 -way analysis of variance, $P<.0001$ ) (Figure 3 ).

Most babies less than 29 weeks' gestation had more than 1 CVC placed during their admission, with the median number of lines placed in this cohort being 2. One infant in this cohort had 5 CVCs placed. After 29 weeks' gestation, most infants had only 1 CVC placed. Compared with baseline, there was no difference in the number of CVCs per patient either overall or when analyzed per gestational age cohort.

\section{Infection}

There were 15 positive blood cultures with clinical signs of infection in the intervention period: 4 were early-onset and

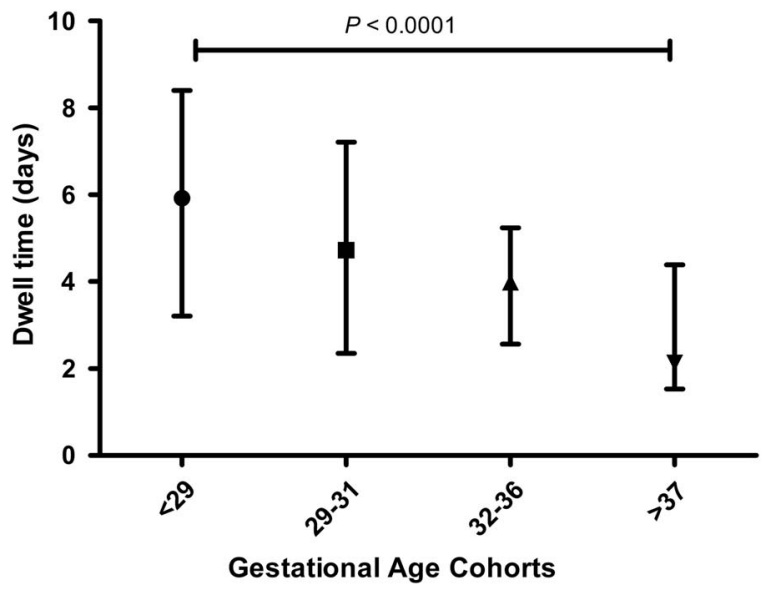

FIGURE 3. Reduction in central venous catheter dwell time in the intervention period across gestational age cohorts.

11 were late-onset sepsis. Of the 11 with culture-positive lateonset sepsis, 3 were CLABSI, all with a pure growth of coagulase-negative Staphylococcus. One other patient had a CVC in situ at the time of deterioration but had a diagnosis of necrotizing enterocolitis with perforated bowel and blood culture grew Enterococcus faecalis. The remaining patients did not have a CVC in situ within 48 hours of bloodstream infection. At baseline, there were 22 bloodstream infections. One of these infections was an early-onset infection, confirmed from blood cultures of samples collected at birth with the same organism isolated in the mother. Another was in a baby without a central line following laser therapy for retinopathy of prematurity. The remaining 20 infections were CLABSI. Of 20 infections, 19 were caused by coagulasenegative Staphylococcus and 1 by Escherichia coli.

Overall there was a significant decrease in CLABSI rates from 8.5 per 1,000 central line-days to 2.3 per 1,000 central line-days (Fisher exact test, $P=.004$ ) (Table 3 ). Three $(1.2 \%)$ of 260 CVC were implicated in infection compared with 20 (5.7\%) of 353 CVC in the baseline data. The run chart of total CLABSI over time is shown in Figure 4 and was extended post hoc to include 2014-2015 data to highlight continued reduced CLABSI rates. 
тавLE 3. Central Line-Associated Infection Rates

\begin{tabular}{|c|c|c|c|c|c|c|}
\hline$\underline{\text { Variable }}$ & \multicolumn{2}{|c|}{ Baseline $(n=214)$} & \multicolumn{2}{|c|}{ Intervention $(n=162)$} & $P$ & $\begin{array}{l}\text { Relative risk } \\
(95 \% \mathrm{CI})\end{array}$ \\
\hline$<29$ weeks & 13.4 & $13 / 966$ & 1.89 & $1 / 530$ & .009 & $0.16(0.02-1.1)$ \\
\hline$<32$ weeks & 11.4 & $19 / 1,664$ & 1.1 & $1 / 946$ & .0003 & $0.11(0.02-0.8)$ \\
\hline PCVC & 11.5 & $19 / 1,653$ & 1.2 & $1 / 820$ & $<.0001$ & $0.1(0.01-0.65)$ \\
\hline
\end{tabular}

NOTE. CL, central line; CLABSI, central line-associated bloodstream infection; N/A, not applicable; PCVC, peripherally inserted central venous catheter; UVC, umbilical venous catheter.

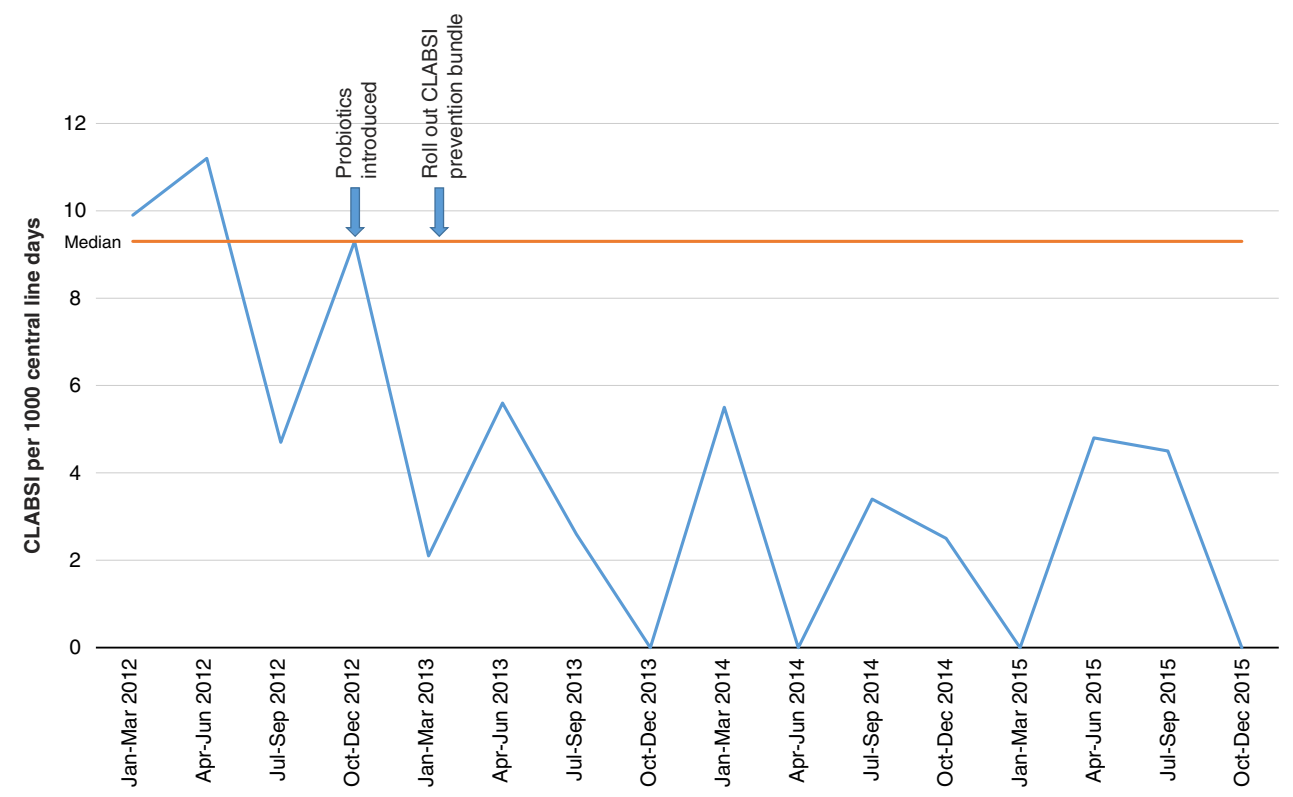

FIGURE 4. Run chart of central line-associated bloodstream infections (CLABSI) per 1,000 central line-days $2012-2015$.

Of interest, 2 of the 3 CVCs in the intervention group that caused clinical sepsis were UVCs, both in infants between 32-34 weeks' gestation. There was only 1 CLABSI seen related to a PCVC, which was in an infant of 25 weeks' gestation who had 5 PCVC in total. There were too few CLABSI in the intervention cohort to perform any further analysis.

\section{I S C USS I O N}

The major findings of this study are that adoption of a coordinated training, education, and review program in a busy Australian neonatal unit has resulted in change in clinical practice, and that this behavioral change translated into significant reductions in clinical complications associated with CVC use. The reductions in the number of CVCs inserted, CVC dwell time, and CLABSI rates provide further data in support of CLABSI prevention intervention bundles.
The reduction of healthcare-associated infection is a nationally important issue, and we have demonstrated the substantial impact that can be made within a single unit with a bundle of interventions.

The intervention package is generalizable to many NICUs in resource-rich countries. Although analyzed retrospectively, data are entered prospectively, infections are coded by the clinical team and the hospital microbiologist, and data are validated by a dedicated audit officer.

Limitations of this study include the relatively small data set, particularly regarding the number of CLABSI. The reduction in infection is, however, in keeping with similar infection prevention bundles in larger studies. ${ }^{20,21}$ It is not a randomized trial and therefore is prone to bias. However, the demographic characteristics of infants in the baseline and intervention groups showed no significant differences. There was no measurement of compliance to the bundle, and in consequence we 
cannot confirm that the interventions described were adhered to and are directly responsible for the reduction in CLABSI. Documentation in medical records has been insufficient to provide other relevant process outcomes. There has been a reduction in sepsis statewide, which has been attributed to a focus on timely data feedback and quality improvement. ${ }^{23}$ Therefore, it is possible that the reduction in CLABSI seen in this study is a reflection of the general trend seen across the state. However, our hospital has shown the largest and most consistent decrease in infection rates statewide ${ }^{23}$ and was the first to implement a bundle of interventions using a structured education program. Ongoing audit will be necessary to document sustained effects. We acknowledge that the introduction of probiotics is a potential confounder. It is possible that both by a direct beneficial effect of probiotics on the immune system ${ }^{31}$ and by reducing times to full feeds, ${ }^{32}$ probiotics may have reduced CLABSI in vulnerable infants by reducing the need for or dwell time of central lines.

CVCs are necessary in many infants, in particular in preterm infants who may take prolonged periods to tolerate full enteral feeds. However, median dwell time of CVCs was 4.4 days overall and just 2.1 days in term infants. There are a number of clinical factors other than time to reach enteral feeds that may influence the decision to insert a CVC, such as difficult peripheral venous access, or the infusion of hyperosmolar or other irritant fluids or medications. The short dwell time seen in this study may reflect a high rate of CVC-related complications and subsequent reinsertion, although the reasons for insertion and removal of CVCs are not routinely collected in our unit. The short dwell time suggests that at least some CVCs were not necessary. CVC dwell time was significantly shorter in infants at least 31 weeks' gestation in the intervention group than at baseline. This may be a consequence of the policy both to avoid CVCs, utilizing peripheral venous access instead, and to remove CVCs at the earliest opportunity, especially in those who are likely to tolerate and reach full feeds more quickly.

There were only 3 CLABSI in the intervention group and consequently too few to perform adjusted analysis or comment further upon the associations or impacts of the CLABSI seen in the intervention period. Only 1 PCVC was related to CLABSI in 1 year of data collection, in a 25 -week infant. However, 2 UVCs were implicated in CLABSI, both in more mature babies. This contrasts with the baseline data, in which most cases of CLABSI were related to PCVC use. This may reflect the emphasis that has been placed on PCVC insertion and maintenance, with a focus on the smallest and most vulnerable infants. Ongoing quality improvement in this unit now focuses on both UVC and PCVC, and the education program emphasizes the importance of the maintenance and insertion bundles in all infants with central lines, regardless of gestation or line type. Further regular audit is important to continue to identify areas of change and should measure compliance to interventions.

Although global incidence of NICU CLABSI is decreasing, ${ }^{7,33}$ central line infection still represents a major risk at an individual level and contributes significantly to length of hospital stay and associated costs. As incidence decreases, it is becoming more important to tackle CLABSI and institute best practices via neonatal networks, such as the Australia and New Zealand Neonatal Network or the Vermont Oxford Network. Walshe demanded that "our expectations of the evidence base for [quality improvement] methodologies should be on a par with our expectation in relation to other forms of healthcare interventions." ${ }^{34(p .153)}$ These networks offer the potential to replicate successful education programs and perform robust interventional studies in this area of quality improvement.

\section{ACKNOWLEDGMENTS}

Financial support. None reported.

Potential conflicts of interest. Both authors report no conflicts of interest relevant to this article.

Address correspondence to Rowena McMullan, FRACP, Department of Newborn Care, Royal Prince Alfred Women and Babies, Missenden Rd, Camperdown, NSW 2050, Australia (rowenamcm@gmail.com).

\section{REFERENCES}

1. Janes M, Kalyn A, Pinelli J, Paes B. A randomized trial comparing peripherally inserted central venous catheters and peripheral intravenous catheters in infants with very low birth weight. J Pediatr Surg 2000;35:1040-1044.

2. Ainsworth SB, Clerihew L, McGuire W. Percutaneous central venous catheters versus peripheral cannulae for delivery of parenteral nutrition in neonates. Cochrane Database Syst Rev 2007:CD004219.

3. O'Grady NP, Alexander M, Burns LA, et al. Guidelines for the prevention of intravascular catheter-related infections. Am J Infect Control 2011;39:S1-S34.

4. Aly H, Herson V, Duncan A, et al. Is bloodstream infection preventable among premature infants? A tale of two cities. Pediatrics 2005;115:1513-1518.

5. Cartwright DW. Central venous lines in neonates: a study of 2186 catheters. Arch Dis Child Fetal Neonatal Ed 2004;89:F504-F508.

6. Milstone AM, Reich NG, Advani S, et al. Catheter dwell time and CLABSIs in neonates with PICCs: a multicenter cohort study. Pediatrics 2013;132:e1609-e1615.

7. Dudeck MA, Weiner LM, Allen-Bridson K, et al. National Healthcare Safety Network (NHSN) report, data summary for 2012, device-associated module. Am J Infect Control 2013;41:1148-1166.

8. Salzman MB, Isenberg HD, Shapiro JF, Lipsitz PJ, Rubin LG. A prospective study of the catheter hub as the portal of entry for microorganisms causing catheter-related sepsis in neonates. J Infect Dis 1993;167:487-490.

9. Salzman MB, Rubin LG. Intravenous catheter-related infections. Adv Pediatr Infect Dis 1995;10:337-368.

10. Shalabi M, Adel M, Yoon E, et al. Risk of infection using peripherally inserted central and umbilical catheters in preterm neonates. Pediatrics 2015;136:1073-1079.

11. Kaplan HC, Lannon C, Walsh MC, Donovan EF, Ohio Perinatal Quality Collaborative. Ohio statewide quality-improvement collaborative to reduce late-onset sepsis in preterm infants. Pediatrics 2011;127:427-435. 
12. Stoll BJ, Hansen N, Fanaroff AA, et al. Late-onset sepsis in very low birth weight neonates: the experience of the NICHD Neonatal Research Network. Pediatrics 2002;110:285-291.

13. Verstraete E, Boelens J, De Coen K, et al. Healthcare-associated bloodstream infections in a neonatal intensive care unit over a 20-year period (1992-2011): trends in incidence, pathogens, and mortality. Infect Control Hosp Epidemiol 2014;35:511-518.

14. Schlapbach LJ, Aebischer M, Adams M, et al. Impact of sepsis on neurodevelopmental outcome in a Swiss National Cohort of extremely premature infants. Pediatrics 2011;128:e348-e357.

15. Stoll BJ, Hansen NI, Adams-Chapman I, et al. Neurodevelopmental and growth impairment among extremely low-birthweight infants with neonatal infection. JAMA 2004;292: 2357-2365.

16. Payne NR, Carpenter JH, Badger GJ, Horbar JD, Rogowski J. Marginal increase in cost and excess length of stay associated with nosocomial bloodstream infections in surviving very low birth weight infants. Pediatrics 2004;114:348-355.

17. Australian Commission on Safety and Quality in Healthcare (ACSQHC). Improvement guide standard 3: preventing and controlling healthcare associated infections. ACSQHC website. http://www.safetyandquality.gov.au/wp-content/uploads/2012/10/ Standard3_Oct_2012_WEB.pdf. Published 2012. Accessed February 19, 2016.

18. Pronovost $\mathrm{P}$, Needham $\mathrm{D}$, Berenholtz $\mathrm{S}$, et al. An intervention to decrease catheter-related bloodstream infections in the ICU. N Engl J Med 2006;355:2725-2732.

19. Blot K, Bergs J, Vogelaers D, Blot S, Vandijck D. Prevention of central line-associated bloodstream infections through quality improvement interventions: a systematic review and metaanalysis. Clin Infect Dis 2014;59:96-105.

20. Schulman J, Stricof R, Stevens TP, et al. Statewide NICU centralline-associated bloodstream infection rates decline after bundles and checklists. Pediatrics 2011;127:436-444.

21. Bizzarro MJ, Sabo B, Noonan M, et al. A quality improvement initiative to reduce central line-associated bloodstream infections in a neonatal intensive care unit. Infect Control Hosp Epidemiol 2010;31:241-248.

22. Greenhalgh M, Gordon A. An audit of central line use and associated blood stream infections in a tertiary NICU. In: Program and abstracts of Perinatal Society of Australia and New Zealand 18th Annual Congress; April 6-9, 2014; Perth, Australia.

23. Shein DS; SPRING Group. The Sepsis Prevention in NICUs Group (SPRING) project: reduction of late onset neonatal infection through collaboration. In: Program and abstracts of Perinatal Society of Australia and New Zealand 18th Annual Congress; April 6-9, 2014; Perth, Australia.

24. Australasian Healthcare Facility Guidelines (AusHFG). Intensive care-neonatal/special care nursery. AusHFG website. https:// healthfacilityguidelines.com.au/hpu/intensive-care-neonatal-specialcare-nursery-0. Accessed May 22, 2016.

25. Ontario Agency for Health Protection and Promotion (Public Health Ontario), Provincial Infectious Diseases Advisory Committee. Best practices for infection prevention and control in perinatology, in all health care settings that provide obstetrical and newborn care. Public Health Ontario website. https://www. publichealthontario.ca/en/eRepository/IPC\%20in\%20Perinatology_ ENGLISH_Final_2012-05-25\%5B1\%5D.pdf. Published 2012. Revised 2015. Accessed February 24, 2016.

26. Hill DA. SCORPIO: a system of medical teaching. Med Teach 1992;14:37-41.

27. Vaughan JI, Jeffery HE, Raynes-Greenow C, et al. A method for developing standardised interactive education for complex clinical guidelines. BMC Med Educ 2012;12:108.

28. SEA-URCHIN Project. South-East Asia-Using Research for Change in Hospital Acquired Infection in Neonates. SEA-URCHIN project website. http://www.seaurchinproject.org/. Published 2011. Accessed February 19, 2016.

29. World Health Organization (WHO). Five moments for hand hygiene. WHO website. http://www.who.int/gpsc/tools/Five_ moments/en/. Published 2015. Accessed February 19, 2016.

30. Dudeck MA, Horan TC, Peterson KD, et al. National Healthcare Safety Network report, data summary for 2011, deviceassociated module. Am J Infect Control 2013;41:286-300.

31. Rao SC, Athalye-Jape GK, Deshpande GC, Simmer KN, Patole SK. Probiotic supplementation and late-onset sepsis in preterm infants: a meta-analysis. Pediatrics 2016;137:1-16.

32. Athalye-Jape G, Deshpande G, Rao S, Patole S. Benefits of probiotics on enteral nutrition in preterm neonates: a systematic review. Am J Clin Nutr 2014;100:1508-1519.

33. Jaggi N, Rodrigues C, Rosenthal VD, et al. Impact of an international nosocomial infection control consortium multidimensional approach on central line-associated bloodstream infection rates in adult intensive care units in eight cities in India. Int J Infect Dis 2013;17:e1218-e1224.

34. Walshe K. Pseudoinnovation: the development and spread of healthcare quality improvement methodologies. Int J Qual Health Care 2009;21:153-159. 